\title{
Superhydrophobic Light Alloy Materials with Corrosion-Resistant Surfaces
}

\author{
Xiaowei XUN, Rili ZHU, Jiaojiao DONG, Ting PAN, Meiling ZHONG, Richao ZHANG, Dongmian ZANG*
}

School of Materials Science and Engineering, East China Jiaotong University, Nanchang 330013, P. R. China

*Corresponding Author: Dongmian ZANG, School of Materials Science and Engineering, East China Jiaotong University, Nanchang 330013, P. R. ChinaE-mail address: zdmian@iccas.ac.cn

\begin{abstract}
:
Metals and their alloys are irreplaceable engineered materials showing great importance in our society. Light alloy materials (i.e., $\mathrm{Mg}, \mathrm{Al}, \mathrm{Ti}$, and their alloys) have tremendous application potential in the aerospace, automotive industries, and biomedical fields for they are lighter and have excellent mechanical properties. The corrosion of light alloys is ubiquitous and greatly restricts their utilization. Inspired by the natural anti-water systems, many new designs and conceptions have recently emerged to create artificial superhydrophobic surfaces with great potential for corrosion resistant of light alloy. This review firstly introduces the concept of superhydrophobicity and strategies of producing superhydrophobic surfaces to inhibit the corrosion of light alloys. In addition, we elaborate the durability of superhydrophobic light alloy materials for commercial and industrial applications, and present their anticorrosion mechanism in the corrosive media.
\end{abstract}

Keywords: light alloys; superhydrophobicity; corrosion resistance; mechanical durability

\section{Introduction}

$\mathrm{Mg}, \mathrm{Al}$, and $\mathrm{Ti}$ based alloys are light alloys materials, showing tremendous application potential for the aerospace, automotive industries, and biomedical engineering owing to their light weight, good ductility, and favorable specific strength ${ }^{[1-3]}$. However, the corrosion of light alloy is ubiquitous and imposes negative effect on the global economy and environment, which greatly restricts their utilization ${ }^{[4,5]}$. Constructing a protective coating on the alloy surface is a common anti-corrosion strategy to isolate the substrate from corrosive liquids. There are so many techniques to prepare corrosion resistant coating for protecting the alloy materials from corrosion factors in the environment, such as organic coating ${ }^{[6]}$, inorganic coating $^{[7]}$, and other composite coating ${ }^{[8]}$. However, most of the coatings are permeable in the corrosion media containing $\mathrm{Cl}^{-}$leading to the corrosion failure of the substrate. Therefore, developing the robust waterproof coatings on light alloy surfaces can be a more effective mothed to protect the light alloy materials from corrosion.

Inspired by the superhydrophobic surfaces in nature, artificial superhydrophobic coatings have been extensively developed due to its unusual properties including self- cleaning, anti-fogging, anti-icing, and anti-biofilm ${ }^{[9]}$. Superhydrophobic surface with hierarchical structure and low surface energy significantly reduce the contact area between water and substrate showing little proneness to water. As such, constructing superhydrophobic metallic surfaces has been an effective strategy to prevent the alloys from being corroded ${ }^{[10]}$. Unlike traditional corrosionresistant coating, superhydrophobic coatings not only provide a barrier between water and alloy material surfaces, but also reduce the residence of excess corrosion solution on the alloy surfaces greatly enhancing capabilities of corrosion protection $^{[11]}$. Superhydrophobic coatings have been successfully developed as protective films on the surfaces of light alloy materials.

This review briefly introduces the superhydrophobic surfaces in nature and presents the fundamental theories on superhydrophobicity and adhesion behavior. Then, we summarize the strategies for preparing superhydrophobic surfaces on light alloys, and suggest the corrosion protection mechanism for superhydrophobic metallic surfaces in corrosive solution and humid environment. Finally, we elaborate the durability of superhydrophobic corrosion-resistant light alloy materials. 


\section{Superhydrophobicity}

The wettability of solid surface is important in many fields, which is governed by surface chemistry as well as surface architecture. In general, the superhydrophobicity as one of the special wetting phenomena on a certain solid surface, is characterized by apparent water contact angle (CA) greater than $150^{\circ[12]}$. The artificial superhydrophobic surface is attractive and has been applied in various fields. Bioinspired approaches are necessary to well understand and create superhydrophobic surfaces ${ }^{[13,14]}$.

\subsection{Natural Superhydrophobicity}

There are lots of insects, plants, and animals with superhydrophobic surfaces in nature, and the superhydrophobicity is a remarkable superiority for these species to survive and evolve in their surroundings ${ }^{[15-17]}$. Superhydrophobic surfaces in nature can be divided into two types. One is a self-cleaning superhydrophobic surface with ultralow water adhesion such as lotus leaf. In 1997, Barthlott and Neinhuis discovered the superhydrophobic selfcleaning property of the lotus attributed to the microscale papillae incorporated into hydrophobic epicuticular wax ${ }^{[18]}$ (figure 1a). Subsequently, many scientists deeply studied the self-cleaning superhydrophobic phenomenon of lotus leaf, and found that the micro/nanoscale hierarchical structure and hydrophobic epicuticular wax responsible for the self-cleaning property ${ }^{[19]}$.

The other is the high-adhesion superhydrophobic surface represented by rose petal (figure $1 \mathrm{~b}$ ). Compared to the self-cleaning lotus leaves, rose petals usually keep the spherical water droplet sticking on their surfaces. As such, the small water droplets are prone to pinning on the surface, while the larger ones roll off like raindrops. To reveal the origin of this strong adhesion on the superhydrophobic surface, Feng et al. studied the microstructure of the rose petal $^{[20]}$. They found that the rose petal surface consists of many micro-papillae with a sea of nanoscale creases at the top of each micro-papilla. This superhydrophobic surface with high CA hysteresis could be illuminated by a impregnating Cassie state: water penetrating into the micro-papillae, and air gaps retaining in the nanoscale folds.

\subsection{Wetting and superhydrophobic}

Over the last few decades, scientists and engineers have intensely studied the wettability of solid surfaces and physical interactions between the solid and liquid. Young equation, Wenzel equation, and Cassie-Baxter equation are the principal equations in this field ${ }^{[21]}$. Young equation is the starting point for wetting state resulting from the equilibrium of interface tension along solid-liquid-vapor interfaces ${ }^{[22]}$. In this context, when the surface tension of water and its environments (usually air) are not changed, decreasing the surface tension of a solid material leads to increasing the static CA. Based on Wenzel equation, making roughness will increase both the hydrophobicity and hydrophilicity ${ }^{[23]}$. If the CA of the surface is greater than $90^{\circ}$, hydrophobicity is improved by roughness. As such, the surface structure is a critical factor for fabricating superhydrophobicity ${ }^{[24]}$. In 1944, Cassie modified the theory of wetting by introducing the concept of area fraction and explain the effect of surface roughness on the apparent CA and the improved hydrophobicity and hydrophilicity ${ }^{[25]}$. In the Cassie state, as the surface roughness is increased, the amount of air trapped in the grooves underneath the liquid is increased and thus the liquid cannot penetrate into the pores due to the trapped air.

Based on the existing wetting theory and superhydrophobic phenomena in nature, several superhydrophobic models have been proposed, which provide theoretical support for the preparation of superhydrophobic materials with specific functions ${ }^{[12]}$. As to the superhydrophobic surface with strong adhesion to water, the surface wetting state is Wenzel state. In Wenzel state (figure 1c), water droplets and substrates are wetcontacted and the water droplet is sticking on the substrate surface, causing a high CA hysteresis. In this context, when the surface is turned upside down, the water droplet can not drop down from the surface, but adhere to the surface, like a water droplet on a rose petal, this unique wetting state is called the "petal" state. Measuring the difference between the advancing CA and the receding CA or directly measuring the sliding angle (SA) can illustrate the CA hysteresis, which is the reflection on the water adhere to the solid surface. Additionally, as shown in figure 1d, as the water droplets contact part of the rough structure, but not contact the substrate, this is considered as a transitional state between Wenzel and Cassie state.

However, the wetting state of superhydrophobic surface with low adhesion force to water is different and has two main types. In Cassie state (figure 1e), the water droplets hardly wet contact on solid surfaces and can roll well off substrates resulting from air trapped underneath the water droplets. In the case, the small SA greater than $10^{\circ}$ can illuminate the wetting functional in Cassie state but not Wenzel state. In addition, the "lotus" state is a special wetting example in Cassie state, for a lotus leaf has the self-cleaning property with micro/nanoscaled hierarchical structures (figure 1f).

According to biological inspiration and the previous wetting theory, there are two ways to prepare superhydrophobic surfaces: one is to roughen the surface of low-surface-energy materials (hydrophobic materials), and the other is to modify the rough surface with low-surfaceenergy materials. While chemical composition and surface roughness are both two main factors to govern surface wettability, the most importance is making rough surface with micro-nano hierarchical structure for constructing the artificial superhydrophobic surface with low adhesion to water. 
(a) Lotus leaf

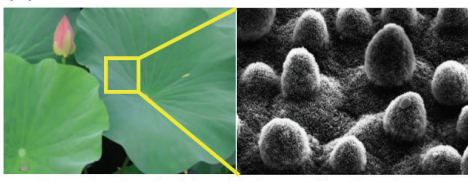

Superhydrophobic with low adhesion force

(c)

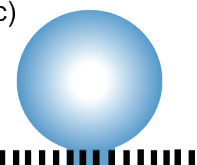

IIIIIIIII

Wenzel's state (d)

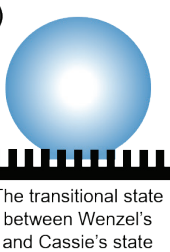

(b) Roses petals

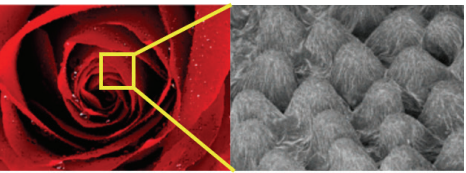

Superhydrophobic with high adhesion force

(e)

IIIIIIIII)

Cassie's state (f)

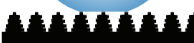

"Lotus" state

Figure 1. Natural superhydrophobic surfaces and their microstructures. (a) Lotus leaf show the superhydrophobic surface with ultralow adhesion due to randomly distributed micro-papillae covered by nanostructures ${ }^{[13]}$. Copyright 2008 Royal Society of Chemistry. (b) Red rose petals exhibit superhydrophobicity with strong adhesion and structural color resulting from periodic arrays of micro-papillae covered by nanofolds ${ }^{[20]}$. Copyright 2008 American Chemical Society. Different states of superhydrophobic surfaces: (c) Wenzel state, (d) the transitional superhydrophobic state between Wenzel and Cassie state, (e) Cassie superhydrophobic state, (f) the "lotus" state (a special case of Cassie superhydrophobic state) ${ }^{[12]}$.

\subsection{Fabrication of superhydrophobic surfaces on light alloy}

For hydrophilic surface of light alloys, the strategy of constructing superhydrophobic surface is to construct a roughness with micro-nano hierarchical structure on the surface of light alloys (There are two main ways to construct roughness, one is bottom-up, that is to prepare a rough coating on the light alloy surface; the other is top-down by making rough structure by physicochemical method on light alloy), and then to reduce the surface energy to achieve superhydrophobic by chemical modification (long alkyl chain thiols, alkyl or fluorinated organic silanes, perfluorinated alkyl agents, long alkyl chain fatty acids, PDMS based polymers, or their combinations) (figure 2). Of course, these two steps can also be synthesized in one step. As such, various fabrication methods including physical, chemical, and combination of physical and chemical methods have been utilized to obtain superhydrophobic light alloy materials. Here, in this section, we will summarize the progress in the preparation of superhydrophobic light alloy materials and their corrosion resistant applications.

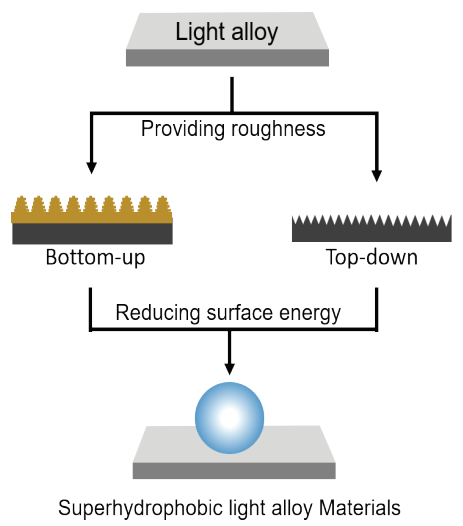

Figure 2. The strategies of producing superhydrophobic light alloy materials.

\subsubsection{Etching}

Etching is the most simple way to prepare rough surfaces on light alloys ${ }^{[26]}$. Etching method includes wet chemical etching, ion etching, plasma etching, and photolithography. Wet chemical etching is very common method for light alloys by immersing in chemical etchants and forms the micro/nanostructure surface with high roughness ${ }^{[27]}$. Then, the superhydrophobic light alloy material can be obtained by low surface energy film self-assemblied on the etching surface. Zang et al. etched Al sheets to achieve the high roughness by using $\mathrm{HCl}$ and then modified with stearic acid in N,N'-dimethylformamide (DMF)/water mixture $^{[28]}$. The obtained $\mathrm{Al}$ alloy surface exhibited an excellent superhydrophobicity with high water CA $167.3^{\circ}$ and corrosion resistant property. However, the chemical etching was carried out by using of various corrosive acids, which is dangerous and not environmentally-friendly ${ }^{[29,30]}$.

Recently, Photolithography is considered to be one of the most effective methods to fabricate a controllable surface roughness on the light alloy surface. Boinovich et al. reported a new efficient method based on nanosecond laser treatment for fabricating a superhydrophobic surface on $\mathrm{Al}$ alloys $^{[31]}$. The results indicated that the surface roughness of $\mathrm{Al}$ alloy can be significantly increased and the morphology can be controlled. After laser etching, the surface of corrugations and grooves is a kind of fibrous and spherical oxide composed of nanoparticles. In addition, a thick oxide film with high roughness covered by nanoparticles will be formed after several stages of melting and solidifying (figure 3). The etched surface can chemically absorb a hydrophobic agent to obtain the superhydrophobic $\mathrm{Al}$ alloy surface with corrosion resistance. Moreover, there are reported strategy to fabricate superhydrophobic Al-Mg alloys with excellent mechanical and chemical properties by combining of functional nanoengineering and nanosecond laser texturing ${ }^{[32]}$. 

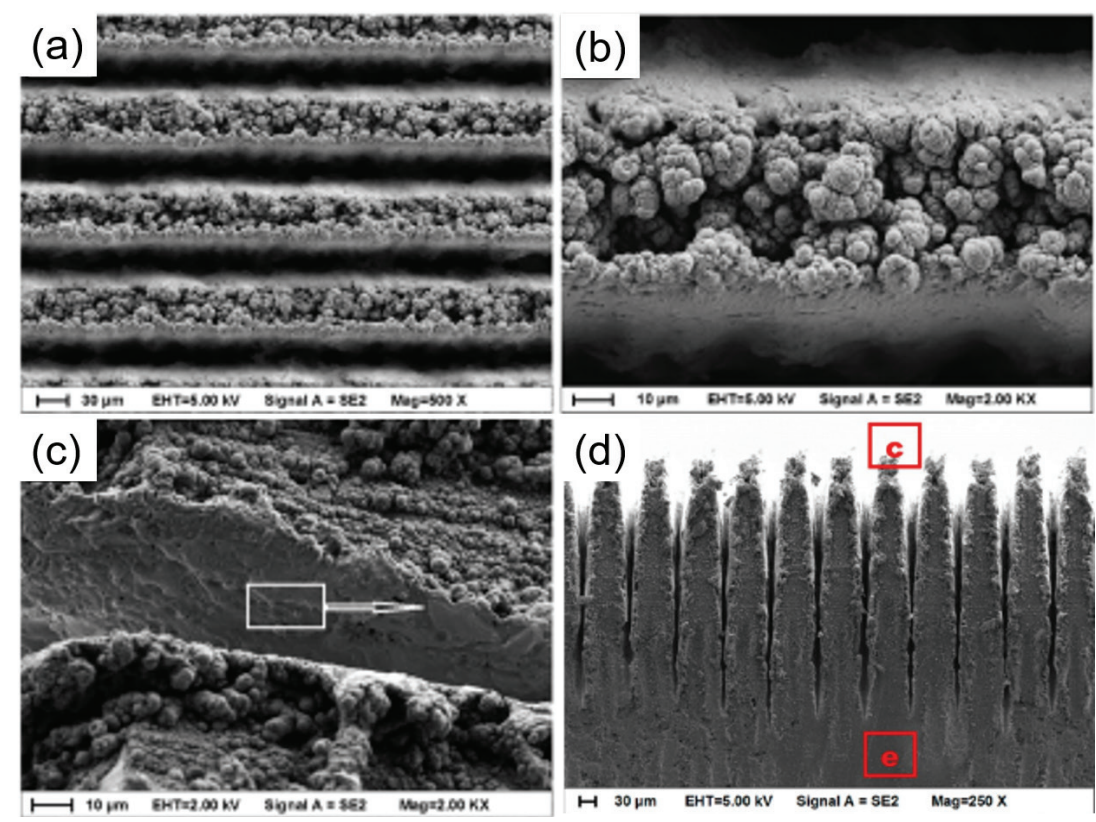

Figure 3. The superhydrophobic oxidized surface fabricated by photolithography on Al alloy ${ }^{[31]}$. The SEM images of the surface texture obtained by many-fold laser treatment $(a, b)$ top view, $(c, d)$ Side view. Scale bars are $(a, d) 30 \mu m,(b, c)$ $10 \mu \mathrm{m}$. Copyright 2015 American Chemical Society.

\subsubsection{Solution immersion}

The solution immersion method is a convenient method for the growth of inorganic coating with rough micronanostructures on the surface of metal materials, which is inexpensive, environmentally-friendly, and easy to carry out $^{[33,34]}$. Qu et al. reported a solution immersion method for fabricating the superhydrophobic surfaces on different metals in a mixed-solution system ${ }^{[35]}$. The as-fabricated solution immersion superhydrophobic surfaces have long-time stability in corrosive liquids and can withstand various concentrations of salt solution. The excellent superhydrophobic surface is mainly attributed to the micro-nanometer structure made by solution immersion method. Furthermore, a superhydrophobic $\mathrm{Fe}(\mathrm{OH})_{3}$ surface on $\mathrm{Mg}$ alloy made by solution immersion process was reported by Zang et al.. And the superhydrophobicity was obtained by stearic acid modified. The resulting superhydrophobic surface had largely improved corrosion resistance of the $\mathrm{Mg}$ alloy ${ }^{[36]}$. Liang et al. immersed the $\mathrm{Mg}$ alloy into $\mathrm{CuCl}_{2}$ solution and stearic acid ethanol solution to fabrication a superhydrophobic surface with the CA of $154^{\text {[37] }}$. The superhydrophobic $\mathrm{Mg}$ alloy surface exhibited excellent durability and corrosion resistance property. The solution immersion method is versatile since it can be applied to various metal materials with complex shapes and different sizes. However, the surface defects and coating adhesion force of solution immersion coating still need to addressed $^{[38]}$.

2.3.3 Hydrothermal

Similar to the solution immersion method, the hydrothermal method is another important bottom-up approach for fabrication of superhydrophobic corrosion proctection coatings on light alloys ${ }^{[39]}$. Different from the solution immersion method, the hydrothermal method is used to synthesize the coatings with micro-/nanostructure on the alloy surface under high temperature and/or pressure. In previous literatures, the superhydrophobic light alloy materials with micro-structured oxide or hydroxide layers was obtained by using hydrothermally treated and selfassembly of $1 \mathrm{H}, 1 \mathrm{H}, 2 \mathrm{H}, 2 \mathrm{H}$-perfluorooctyltriethoxysilane (PFOTES $)^{[40]}$. As such, Feng et al. fabricated the superhydrophobic $\mathrm{Mg}$ alloy surface using an environmentfriendly, facile, and cost-effective one-step hydrothermal process $^{[41]}$. The as-prepared superhydrophobic $\mathrm{Mg}$ alloy surface with rough and hierarchical micro-/nanostructure exhibits excellent corrosion resistance and self-cleaning performance (figure 4).

\subsubsection{Electrochemical}

The electrochemical process can be employed to control surfacemorphology oflightalloys, even on verylarge surfaces with various surface morphologies ${ }^{[42]}$. In this context, there are two strategies to fabricate superhydrophobic surface by electrochemical process. The first is the anodization processes, the anodizing method is one of the wellestablished electrochemistry to form the oxide layer with micro-nanoscale roughness on the metal surface, which is used for the preparation of superhydrophobic surface. The obtained anodization superhydrophobic surfaces have strong adhesion between the coating and the substrate and have wide applications ${ }^{[43]}$. Vengatesh et al. reported a superhydrophobic anodic aluminum oxide surface by using anodization process for corrosion protection of $\mathrm{Al}$ alloy ${ }^{[44]}$ (figure 5a). The prepared aluminum anodizing film not only has strong surface adhesion to the substrate, but also is important to fatty acids grafting ensuring the stability of superhydrophobic surface with good corrosion resistance. 
Secondly, the electrodeposition is a bottom-up approach for depositing the metal and/or metal oxide onto various conductive substrates ${ }^{[45,46]}$. It is an effective technique for fabricating high roughness coatings on various substrates, for it can easily form micro-nanostructures by adjusting the electrodeposition parameters. He et al. fabricated the superhydrophobic surfaces with dendritic structures on Ti6Al4V surface by using electrodeposition ${ }^{[47]}$ (figure 5b). The superhydrophobic coating with dendritic hierarchical structure was constructed by electrodepositing and annealing and displaying superhydrophobicity and low adhesion to water because of complex micro/ nanostructures.
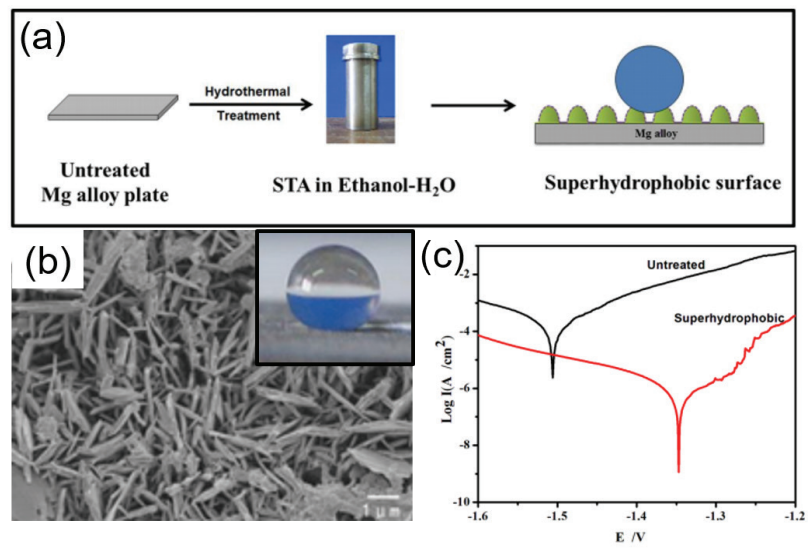

Figure 4. The superhydrophobic surface prepared by hydrothermal process on Mg alloy ${ }^{[41]}$. (a) Schematic of the fabrication of superhydrophobic surface on Mg alloy with one-step hydrothermal process. (b) The SEM images of one-step hydrothermal coatings. (c) Corrosion behaviors of untreated and superhydrophobic surface fabricated by hydrothermal. Copyright 2017 Elsevier.
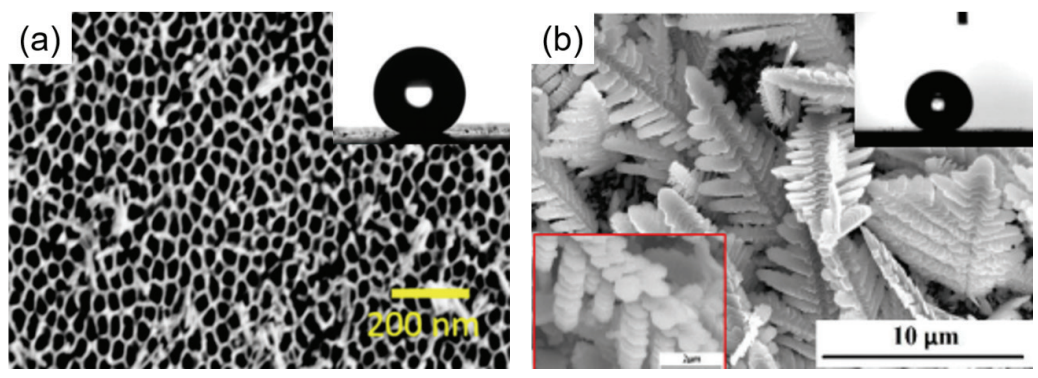

Figure 5. The surface morphologies and wetting behaviors of electrochemical superhydrophobic surface. (a) The SEM image and water $\mathrm{CA}$ of anodization superhydrophobic nanoporous $\mathrm{Al}_{2} \mathrm{O}_{3}$ surface ${ }^{[44]}$. Copyright 2015 American Chemical Society. (b) SEM images of electrodeposition superhydrophobic $\mathrm{Zn} / \mathrm{ZnO} / \mathrm{TiO}_{2}$ surface on surface of Ti6Al4V. The inset is the water CA of corresponding surfaces ${ }^{[47]}$. Copyright 2017 Elsevier.

\section{Durable Superhydrophobic Light Alloy Ma- terials}

\subsection{Robust superhydrophobic light alloy materials}

A myriad of reports have been published on fabricating superhydrophobic light alloy materials with corrosion resistant performance. However, the poor mechanical stability of microscopic surface structure has negative effect on the durability of superhydrophobic surface ${ }^{[48]}$. Mechanical wear on superhydrophobic light alloy materials usually leads to loss of water repellency property and makes the corrosion of the substrate more easily occur. In this context, the importance of superhydrophobic mechanical durability in applications should be addressed ${ }^{[49,50]}$.

As we all know, the stability of hierarchical structure on superhydrophobic surface is critically important to the robustness of superhydrophobic. Such hierarchical structure (submicron-sized nanobumps superimposed over microscale roughness) more durable than nanostructure surface ${ }^{[51]}$. Therefore, it is worth to note that the roughness of superhydrophobic surface should be optimized in the process of preparing superhydrophobic surface to improve its mechanical robustness. Luo et al. reported a superhydrophobic surface which has the robust mechanical durability because of the flexible coral-reeflike hierarchical structure ${ }^{[52]}$ (figure 6). The durability of hierarchical architectures is attributed to the flexible coralreef-like structure constructed by kinetic spray process. And the self-similar manner of the fluorinated wear-resistant porous oxide layer makes the obtained superhydrophobic coating has good water repellency. Based on combination of the above two strategies, the $\mathrm{Cu}$-based superhydrophobic 
coating maintains its superhydrophobicity after various harsh mechanical testings. Particularly, the cushion effect of the coral-reef-like hierarchical structure could greatly minimize the mechanical damage from the environment to prevent the loss of superhydrophobic.

Most of the materials are conventionally hydrophilic, so a hydrophobic coating is required to make a superhydrophobic surface. However, the obtained traditional superhydrophobic surface has a disadvantage that the hydrophobic film has poor interface bonding force and rough structure is unstable, which results in the exposure of hydrophilic material after mechanical abration $^{[48]}$. Then the water sticks to the tops of the abraded surface and the superhydrophobicity is lost. An effective way to deal with the above problem is to prepare the roughness on light alloys by using robust hydrophobic materials. Peng et al. presented all-organic, flexible, and multi-fluorination superhydrophobic nanocomposite coatings, which has strong substrate adhesion and excellent mechanical and chemical robustness ${ }^{[53]}$ (figure 7 ). The robustness of the superhydrophobic nanocomposite coatings was resultant from a namely multi-fluorination strategy. The epoxy resin, perfluoropolyether, and fluoropolymer nanoparticles give rise to the stable hierarchical hydrophobic structure and help the surface energy, flexibility, and robustness to remain. As such, multi-fluorination coatings can retain superhydrophobicity even subjected to mechanical abrasion.

\subsection{Self-healing superhydrophobic light alloy materials}

Natural superhydrophobic surface can sustain their permanent water repellency and withstand damage because of their continuous repairing ability. Therefore, the self-healing superhydrophobic coatings have gained widespread interest, which can self-repair the damage caused by external factors and increase the durability of artificial superhydrophobic surface ${ }^{[54]}$. In addition, the selfhealing superhydrophobic coating can ensure the durability of the hydrophobic property of the coating and protect the light alloy substrate from corrosion. Zhang et al. fabricated a self-healing superhydrophobic coating on $\mathrm{Mg}$ alloy from a eutectic solvent containing $\mathrm{Cr}$ (III) ${ }^{[55]}$ (figure 8 ). The selfhealing superhydrophobic coating was composed of $\mathrm{Cr}_{2} \mathrm{O}_{3}$ hierarchical microstructure and stearic acid modified layer. The smart superhydrophobic coating had shown excellent corrosion resistance in the $\mathrm{NaCl}$ aqueous solution due to the dual corrosion protect function. Compared with the traditional superhydrophobic coating, the second corrosion barrier of self-healing superhydrophobic coating is impressive. When the superhydrophobic coating is destroyed in the corrosion medium, the activated selfhealing ability can repair the damage and maintain the durable corrosion resistance.
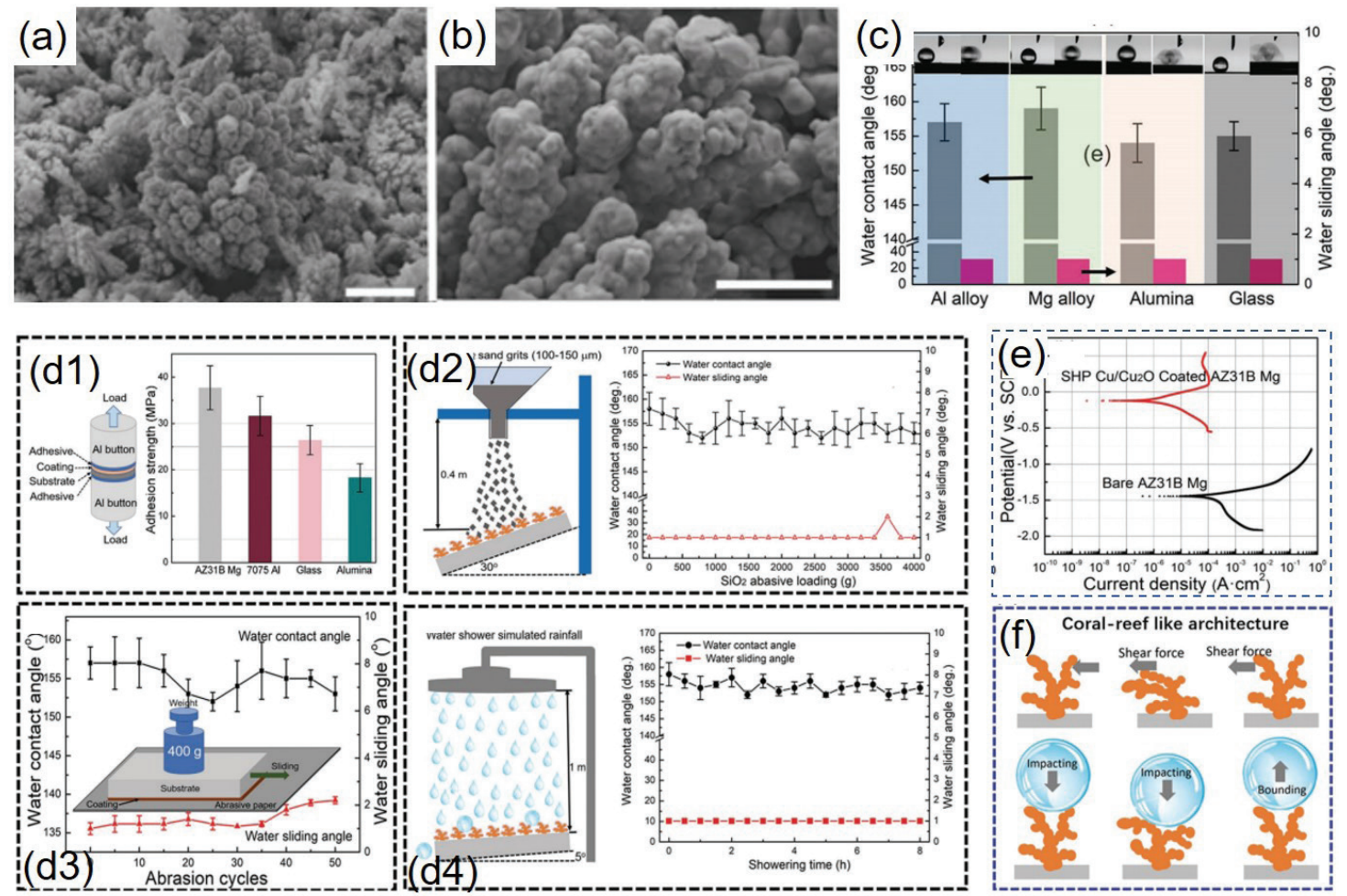

Figure 6. Superhydrophobic surface with a flexible hierarchical rough structure ${ }^{[52]}$ : The surface topography of the flame-oxidized coating (a) and enlarged(b). (c) Superhydrophobicity on various substrates. (d) Mechanical robustness of the $\mathrm{Cu}$-based superhydrophobic coating with flexible hierarchical rough structure. (e) Corrosion resistance test of $\mathrm{Cu}$ based superhydrophobic coating. (f) Schematics showing the responses of flexible microstructure on Cu-based superhydrophobic coating to abration and impact. Copyright 2019 Wiley. 
(a)

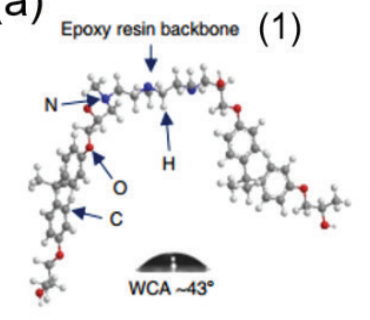

(b)

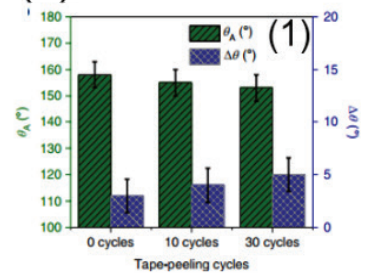

(c)
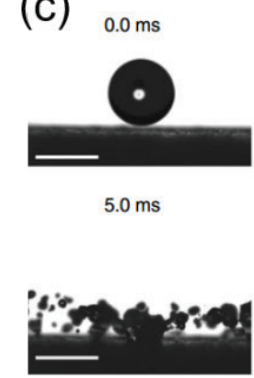

$0.4 \mathrm{~ms}$

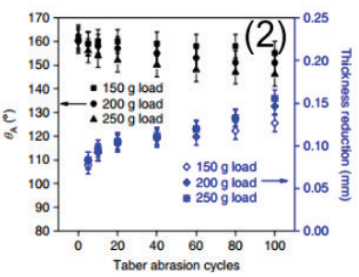

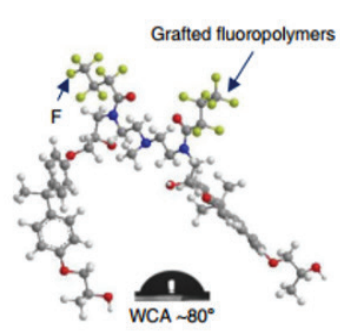

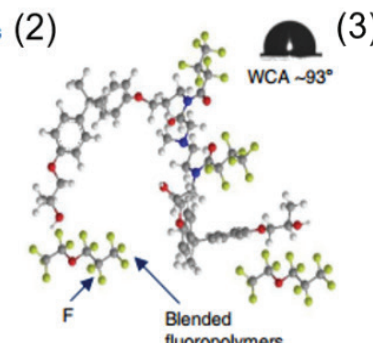

(3)
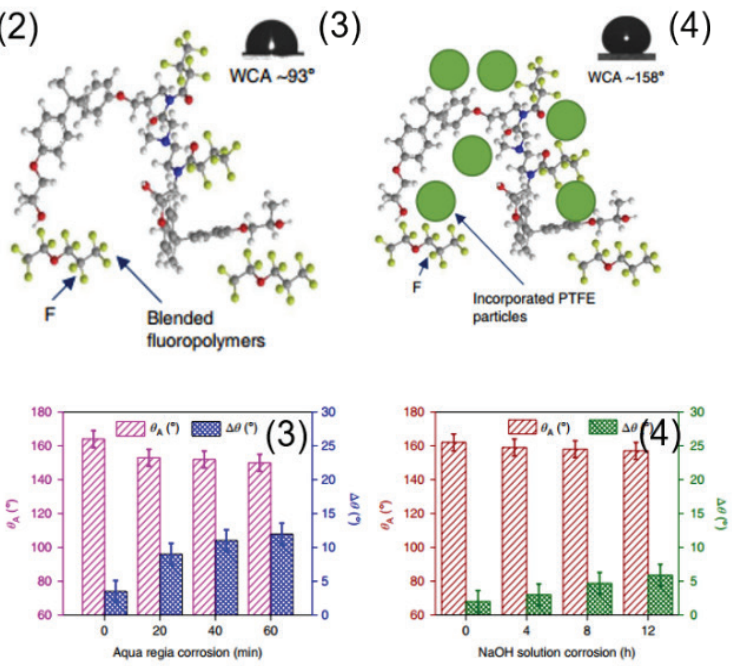

(d)

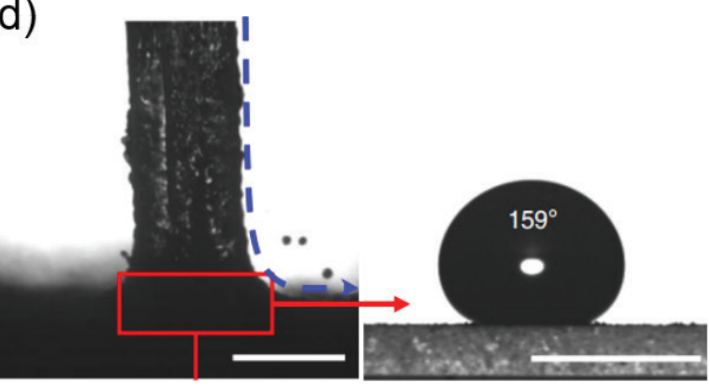

Figure 7. All-organic superhydrophobic coatings ${ }^{[53]}$ : (a) Schematic illustration for the nanocomposite coating with the multi-fluorination strategy. (b) Mechanical robustness and chemical resistance of all-organic superhydrophobic coatings.A high-speed water jet test (c) and a water drop on the all-organic superhydrophobic coatings after water jet (d). Copyright 2018 Nature.

(a)

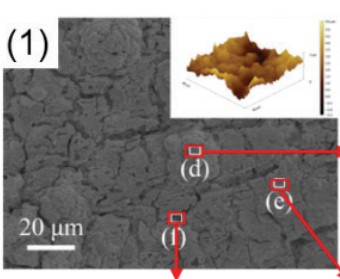

(3)

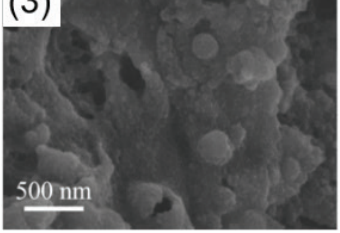

(c) (1) 0 min

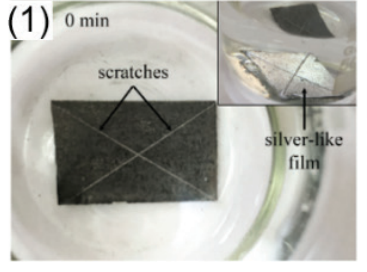

(2)

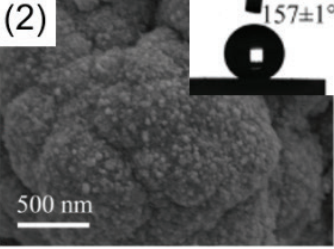

(4)
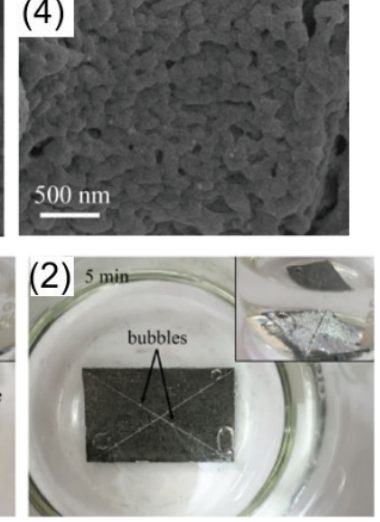

(b)
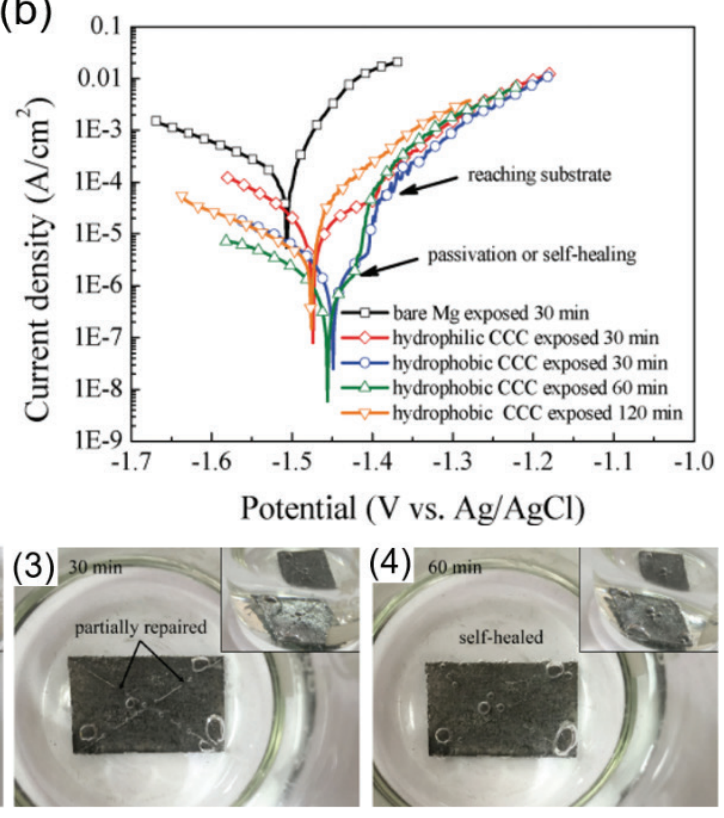

Figure 8. Self-healing superhydrophobic coating on Mg alloy ${ }^{[55]}$ : (a) Surface morphologies and water CA of self-healing superhydrophobic coating. (b) Corrosion resistance of self-healing superhydrophobic coating. (c) The photos of self-healing capability of scratched self-healing superhydrophobic coating on Mg alloy. Copyright 2016 Wiley. 


\section{Application of Superhydrophobic Surfaces for Corrosion Protection}

Metal corrosion is the depletion or destruction caused by the interaction between metal materials and the environment, which changes the original properties and resulting in the loss of its function as engineering materials ${ }^{[56]}$. Humidity, salt, acids, bases, and solvent are all factors causing corrosion of metal and/or its alloys in real environment. When the metal materials are exposed to corrosion environment and contact with those corrosion media, corrosion will take place on the surface and continues to spread rapidly in the materials.

Various surfaces and coating technologies have been proposed to achieve the corrosion resistance on the light alloy surfaces. Superhydrophobic surfaces on light alloy materials have shown remarkable corrosion-resistance in corrosive solution media during the past two decades ${ }^{[57]}$. Superhydrophobic coatings, like other coatings, provide a barrier on the surface of light alloys to effectively isolate the corrosion solution from the substrate. It is noted that superhydrophobic light alloy materials with hierarchical structure surface can trapped more air when immersed in the corrosive liquid, thus reducing the contact area between the corrosive solution and the materials surface and greatly reducing the corrosive media attacked to the substrate. The new effective mechanism for corrosion protection is provided $^{[58,59]}$ (figure 9a). In addition, the self-cleaning properties of the superhydrophobic light alloy materials can also remove the corrosive medium on the surface and further reduce corrosion ${ }^{[60]}$. The superhydrophobic surface with low surface energy played irreplaceable roles in this process. On the other hand, the nanostructure of hierarchical rough structure on superhydrophobic light alloy materials is very important for preventing nanodroplets from pinning when exposed to humid environment. As can be seen in figure $9 \mathrm{~b}$, the condensed droplets are isolated from each other on the hierarchical structure and the electron transfer in the corrosion medium could be impeded, which inhibiting the electrochemical reaction and reducing the possibility of corrosion ${ }^{[61,62]}$.
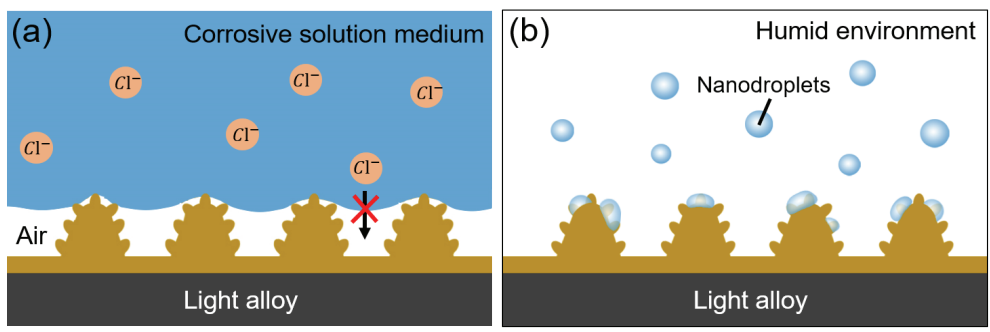

Figure 9. Illustration for corrosion protection of superhydrophobic light alloy materials in a corrosive solution medium (a) and humid environment (b). Copyright 2014 Elsevier.

In addition, different alloys have their own corrosion behaviors because of chemical and electrochemical properties. $\mathrm{Mg}$ based material is an active engineering material and has an aggressive driving force for corrosion. In a nature environment, the $\mathrm{Mg}(\mathrm{OH})_{2}$, films on the surface of $\mathrm{Mg}$ and its alloys was formed, but they are poorly protective ${ }^{[63]}$. As such, it is necessary to fabricate a corrosion resistance coating for $\mathrm{Mg}$ and its alloys without sacrificing the favorable physical and mechanical properties. There are, however, many pores and cracks on the coated $\mathrm{Mg}$ alloy surface, which are difficult to avoid ${ }^{[64]}$. These defects provide a path for the penetration of corrosive medium and accelerate the corrosion of $\mathrm{Mg}$ alloys. Therefore, fabricating water-repellent coatings on $\mathrm{Mg}$ alloys has superiority to other systems for corrosion protection.

In the case of $\mathrm{Al}$, the inherent corrosion resistance of $\mathrm{Al}$ and its alloy is attributed to its continuous surface oxide layer surface, which can significantly prevent internal corrosion of materials. However, the stable oxide layer on $\mathrm{Al}$ can be destroyed and made it extremely vulnerable to corrosion when exposed to strong acid, strong alkaline solutions or solution with aggressive anions. To compensate for the deficiency, preparation of ultralow adhesion superhydrophobic coatings on $\mathrm{Al}$ alloy is a promising technique to prevent the damage of oxide layer and materials corrosion ${ }^{[44]}$. Similarly, Ti and its alloy also have unusual corrosion resistant to alkali, chloride and some strong acids because of the compact oxide film formed spontaneously on surfaces. However, when Ti and its alloy encounter reductive oxygen and HF medium, the corrosion behavior are also arise. Superhydrophobic surfaces on titanium and its alloys can protect the metal materials from corrosion in harsh environment ${ }^{[65]}$.

\section{Summary}

Making bioinspired superhydrophobic coating is an important method to protect light alloy materials from corrosion in previous studies. In this review, we have shown the researching progress in superhydrophobic light alloy materials with corrosion-resistant surfaces. We summarize the strategies of preparing bioinspired superhydrophobic light alloy materials based on the wetting theory. Superhydrophobic coating and air film trapped in hierarchical structure form double anticorrosive barrier, which can greatly reduce the possibility of alloy substrate contacting with corrosive liquid and display superior corrosion resistance performances. Furthermore, mechanical durability is a central concern for superhydrophobic light alloy materials. Several studies 
have addressed this issue by improving the durability and/ or possessing self-healing capability for superhydrophobic materials, which contribute to reduce the surface damage and prolong the lifetime of superhydrophobic surfaces.

Conflict of Interest: No conflict of interest was reported by the authors.

Acknowledgments: This work was supported by the National Natural Science Foundation of China (51863008, 51903084), the Natural Science Foundation of Jiangxi Province (20192BAB203008, 20192BAB206015).

\section{References}

[1] Ovari F, Tomcsanyi L, Turmezey T. Electrochemical study of the pitting corrosion of aluminum and its alloy- I . Determination of critical pitting and protection potentions. Electrochimica Acta 1988; 33(3): 323-326.

[2] Gurrappa I. Characterization of titanium alloy Ti-6Al$4 \mathrm{~V}$ for chemical, marine and industrial applications. Materials Characterization 2003; 51(2-3): 131-139.

[3] Ballerini G, Bardi $U$, Bignucolo $R$, et al. About some corrosion mechanisms of AZ91D magnesium alloy. Corrosion Science 2005; 47(9): 2173-2184.

[4] Devarayan K, Mayakrishnan G, Nagarajan S. Green inhibitors for corrosion of metals a review. Chemical Science Review and Letters 2012; 1(1): 1-8.

[5] Bonita D, Yeojin J, Jeongsoo S, et al. Review on corrosion behavior of metallic materials in room temperature ionic liquids International Journal of Electrochemical Science 2016; 11: 1482-1495.

[6] Voevodin N N, Grebasch N T, Soto W S, et al. An organically modified zirconate film as a corrosionresistant treatment for aluminum 2024-T3. Progress in Organic Coatings 2001; 41: 287-293.

[7] Sidky P S, Hocking M G. Review of inorganic coatings and coating processes for reducing wear and corrosion. British Corrosion Journal 1999; 3: 171-183.

[8] Chou T P, Chandrasekaran C, Limmer S J, et al. Organicinorganic hybrid coating for corrosion protection. Journal of Non-Crystalline Solids 2001; 290: 153-162.

[9] Zhang X, Shi F, Niu J, et al. Superhydrophobic surfaces: from structural control to functional application. Jounal of Materials Chemistry 2008; 18(6): 621-633.

[10] Zhang F, Zhao L, Chen $\mathrm{H}$, et al. Corrosion resistance of superhydrophobic layered double hydroxide films on aluminum. Angewandte Chemie International Edition 2008; 47(13): 2466-2469.

[11] Wang P, Zhang D, Lu Z. Advantage of super-hydrophobic surface as a barrier against atmospheric corrosion induced by salt deliquescence. Corrosion Science 2015; 90: 23-32.

[12] Wang S, Jiang L. Definition of Superhydrophobic States. Advanced Materials 2007; 19(21): 3423-3424.
[13] Su B, Tian Y, Jiang L. Bioinspired interfaces with superwettability: From materials to chemistry. Journal of the American Chemical Society 2016; 138(6): 17271748.

[14] Darmanin T, Guittard F. Recent advances in the potential applications of bioinspired superhydrophobic materials. Journal of Materials Chemistry A 2014; 2(39): 1631916359.

[15] Wang S, Liu K, Yao X, et al. Bioinspired surfaces with superwettability: new insight on theory, design, and applications. Chemical Reviews 2015; 115(16): 82308293.

[16] Zang D, Zhu R, Zhang W, et al. Corrosion-resistant superhydrophobic coatings on Mg alloy surfaces inspired by lotus seedpod. Advanced Functional Materials 2017; 27(8): 1605446.

[17] Lu X, Cai H, Wu Y, et al. Peach skin effect: A quasisuperhydrophobic state with high adhesive force. Science Bulletin 2015; 60(4): 453-459.

[18] Barthlott W, Nieinhuis C. Purity of the sacred lotus, or escape from contamination in biological surfaces. Planta 1997; 202: 1-8.

[19] Feng L, Li S, Li Y, et al. Super-hydrophobic surfaces: Frim natural to artificial. Advanced Materials 2002; 14: 18571860.

[20] Feng L, Zhang Y, XiJ, et al. Petal effect: A superhydrophobic state with high adhesive force. Langmuir 2008; 24: 4114-4119.

[21] Whyman G, Bormashenko E, Stein T. The rigorous derivation of Young, Cassie-Baxter and Wenzel equations and the analysis of the contact angle hysteresis phenomenon. Chemical Physics Letters 2008; 450(4-6): 355-359.

[22] Young T. An essay on the cohesion of fluids. Philosophical transactions of the royal society of London 1805; 95(95): 65-87.

[23] Wenzel R N. Resistance of solid surfaces to wetting by water. Industrial \& Engineering Chemistry 1936; 28(8): 988-994.

[24] Callies $M$, Quéré D. On water repellency. Soft Matter 2005; 1(1): 55-61.

[25] Cassie A B D, Baxter S. Wettability of porous surfaces. Transactions of the Faraday society 1944; 40: 546-551.

[26] Wang $Y$, Gu Z, Xin Y, et al. Facile formation of superhydrophobic nickel coating on magnesium alloy with improved corrosion resistance. Colloids and Surfaces A: Physicochemical and Engineering Aspects 2018; 538: 500-505.

[27] Huang Y, Sarkar D K, Grant Chen X. Superhydrophobic aluminum alloy surfaces prepared by chemical etching process and their corrosion resistance properties. Applied Surface Science 2015; 356: 1012-1024.

[28] Zang D, Zhu R, Zhang W, et al. Stearic acid modified 
aluminum surfaces with controlled wetting properties and corrosion resistance. Corrosion Science 2014; 83: 86-93.

[29] Liu L, Xu F, Ma L. Facile fabrication of a superhydrophobic Cu surface via a selective etching of high-energy facets. The Journal of Physical Chemistry C 2012; 116(35): 18722-18727.

[30] Feng L, Zhang H, Wang Z, et al. Superhydrophobic aluminum alloy surface: fabrication, structure, and corrosion resistance. Colloids and Surfaces A: Physicochemical and Engineering Aspects 2014; 441: 319-325.

[31] Boinovich L B, Emelyanenko A M, Modestov A D, et al. Synergistic effect of superhydrophobicity and oxidized layers on corrosion resistance of aluminum alloy surface textured by nanosecond laser treatment. ACS Appllied Materials \& Interfaces 2015; 7(34): 19500-19508.

[32] Boinovich L B, Modin E B, Sayfutdinova A R, et al. Combination of functional nanoengineering and nanosecond laser texturing for design of superhydrophobic aluminum alloy with exceptional mechanical and chemical properties. ACS Nano 2017; 11(10): 10113-10123.

[33] Liu Y, Lu G, Liu J, et al. Fabrication of biomimetic hydrophobic films with corrosion resistance on magnesium alloy by immersion process. Applied Surface Science 2013; 264: 527-532.

[34] Song J, Lu Y, Huang S, et al. A simple immersion approach for fabricating superhydrophobic $\mathrm{Mg}$ alloy surfaces. Applied Surface Science 2013; 266: 445-450.

[35] Qu M, Zhang B, Song S, et al. Fabrication of superhydrophobic surfaces on engineering materials by a solution-immersion process. Advanced Functional Materials 2007; 17(4): 593-596.

[36] Zang D, Zhu R, Wu C, et al. Fabrication of stable superhydrophobic surface with improved anticorrosion property on magnesium alloy. Scripta Materialia 2013; 69(8): 614-617.

[37] Liang M, Wei Y, Hou L, et al. Fabrication of a superhydrophobic surface on a magnesium alloy by a simple method. Journal of Alloys and Compounds 2016; 656: 311-317.

[38] Zhao L, Liu Q, Gao R, et al. One-step method for the fabrication of superhydrophobic surface on magnesium alloy and its corrosion protection, antifouling performance. Corrosion Science 2014; 80: 177-183.

[39] Zheng T, Hu Y, Zhang Y, et al. Formation of a hydrophobic and corrosion resistant coating on magnesium alloy via a one-step hydrothermal method. Journal of Colloid and Interface Science 2017; 505: 87-95.

[40] Ou J, Hu W, Xue M, et al. Superhydrophobic surfaces on light alloy substrates fabricated by a versatile process and their corrosion protection. ACS Applied Materials \&
Interfaces 2013; 5(8): 3101-3107.

[41] Feng L, Zhu Y, Wang J, et al. One-step hydrothermal process to fabricate superhydrophobic surface on magnesium alloy with enhanced corrosion resistance and self-cleaning performance. Applied Surface Science 2017; 422: 566-573.

[42] Darmanin T, Taffin de Givenchy E, Amigoni S, et al. Superhydrophobic surfaces by electrochemical processes. Advanced Materials 2013; 25(10): 13781394.

[43] Wang D, Wang X, Liu X, et al. Engineering a titanium surface with controllable oleophobicity and switchable oil adhesion. Journal of Physical Chemistry C 2010; 114: 9938-9944.

[44] Vengatesh $P$, Kulandainathan M A. Hierarchically ordered self-lubricating superhydrophobic anodized aluminum surfaces with enhanced corrosion resistance. ACS Appllied Materials \& Interfaces 2015; 7(3): 15161526.

[45] Wang H, Zhu Y, Hu Z, et al. A novel electrodeposition route for fabrication of the superhydrophobic surface with unique self-cleaning, mechanical abrasion and corrosion resistance properties. Chemical Engineering Journal 2016; 303: 37-47.

[46] Zhang B, Zhao X, Li Y, et al. Fabrication of durable anticorrosion superhydrophobic surfaces on aluminum substrates via a facile one-step electrodeposition approach. RSC Advances 2016; 6(42): 35455-35465.

[47] He G, Lu S, Xu W, et al. Durable superhydrophobic $\mathrm{Zn} / \mathrm{ZnO} / \mathrm{TiO}_{2}$ surfaces on $\mathrm{Ti}_{6} \mathrm{Al}_{4} \mathrm{~V}$ substrate with selfcleaning property and switchable wettability. Ceramics International 2018; 44(1): 638-647.

[48] Verho T, Bower C, Andrew P, et al. Mechanically durable superhydrophobic surfaces. Advanced Materials 2011; 23(5): 673-678.

[49] Zhang D, Wang L, Qian H, et al. Superhydrophobic surfaces for corrosion protection: a review of recent progresses and future directions. Journal of Coatings Technology and Research 2015; 13(1): 11-29.

[50] XuelinT,TuukkaV,Robin H.A.R.Movingsuperhydrophobic surfaces toward real-world applications. Science 2016; 352(6268): 142-143.

[51] Li W, Amirfazli A. Hierarchical structures for natural superhydrophobic surfaces. Soft Matter 2008; 4(3): 462 466.

[52] Luo X T, Li C J. Bioinspired mechanically robust metalbased water repellent surface enabled by scalable construction of a flexible coral-reef-like architecture. Small 2019; 15(39): e1901919.

[53] Peng C, Chen Z, Tiwari M K. All-organic superhydrophobic coatings with mechanochemical robustness and liquid impalement resistance. Nature Materials 2018; 17(4): 355-360. 
[54] Xue C-H, Ma J-Z. Long-lived superhydrophobic surfaces. Journal of Materials Chemistry A 2013; 1(13): 41464161.

[55] Zhang J, Gu C, Tong Y, et al. A smart superhydrophobic coating on AZ31B magnesium alloy with self-healing effect. Advanced Materials Interfaces 2016; 3(14): 1500694.

[56] Montemor M. Functional and smart coatings for corrosion protection: a review of recent advances. Surface and Coatings Technology 2014; 258: 17-37.

[57] Vazirinasab E, Jafari R, Momen G. Application of superhydrophobic coatings as a corrosion barrier: $A$ review. Surface and Coatings Technology 2018; 341: 4056.

[58] Cui L-Y, Fang $X-H$, Cao $W$, et al. In vitro corrosion resistance of a layer-by-layer assembled DNA coating on magnesium alloy. Applied Surface Science 2018; 457: 49-58.

[59] Zhang D, Li L, Wu Y, et al. One-step method for fabrication of bioinspired hierarchical superhydrophobic surface with robust stability. Applied Surface Science 2019; 473: 493-499.
[60] Zheng S, Li C, Fu Q, et al. Development of stable superhydrophobic coatings on aluminum surface for corrosion-resistant, self-cleaning, and anti-icing applications. Materials \& Design 2016; 93: 261-270.

[61] Michael N, Bhushan B. Hierarchical roughness makes superhydrophobic states stable. Microelectronic Engineering 2007; 84(3): 382-386.

[62] Wang N, Xiong D. Superhydrophobic membranes on metal substrate and their corrosion protection in different corrosive media. Applied Surface Science 2014; 305: 603-608.

[63] Song G, Atrens A. Understanding magnesium corrosion-A framework for improved alloy Performance. Advanced Engineering Materials 2003; 5(12): 837-858.

[64] Chu Q, Liang J, Hao J. Facile fabrication of a robust superhydrophobic surface on magnesium alloy. Colloids and Surfaces A: Physicochemical and Engineering Aspects 2014; 443: 118-122.

[65] Ou J, Liu M, Li W, et al. Corrosion behavior of superhydrophobic surfaces of Ti alloys in $\mathrm{NaCl}$ solutions. Applied Surface Science 2012; 258(10): 4724-4728. 